\title{
The use of the Psychiatric Electroencephalography Evaluation Registry (PEER) to personalize pharmacotherapy
}

This article was published in the following Dove Press journal:

Neuropsychiatric Disease and Treatment

25 August 2016

Number of times this article has been viewed

\author{
Dan V losifescu' \\ Robert J Neborsky ${ }^{2-4}$ \\ Robert J Valuck ${ }^{5-7}$ \\ 'Adult Psychopharmacology Program, \\ Psychiatry and Neuroscience, Icahn \\ School of Medicine at Mount Sinai, \\ New York, NY, USA; ${ }^{2}$ School of \\ Medicine, University of California, \\ San Diego, CA, USA; ${ }^{3}$ University of \\ California, Los Angeles, CA, USA; \\ ${ }^{4}$ Medical Corps, US Navy, USA; \\ ${ }^{5}$ Pharmacy, Epidemiology, and Family \\ Medicine, University of Colorado, \\ Denver, CO, USA; ${ }^{6}$ Center for \\ Pharmaceutical Outcomes Research, \\ University of Colorado, Denver, CO, \\ USA; ${ }^{7}$ Colorado Consortium for \\ Prescription Drug Abuse Prevention, \\ Denver, CO, USA
}

Correspondence: Dan V losifescu Psychiatry and Neuroscience, Icahn School of Medicine at Mount Sinai, One Gustave L Levy Place, Box 1230 , New York, NY 10029, USA

$\mathrm{Tel}+\mid$ 2I $224 \mid 4480$

Fax + I 2122413354

Email dan.iosifescu@mssm.edu
Purpose: This study aims to determine whether Psychiatric Electroencephalography Evaluation Registry (PEER) Interactive (an objective, adjunctive tool based on a comparison of a quantitative electroencephalogram to an existing registry of patient outcomes) is more effective than the current standard of care in treatment of subjects suffering from depression.

Patients and methods: This is an interim report of an ongoing, 2-year prospective, randomized, double blind, controlled study to evaluate PEER Interactive in guiding medication selection in subjects with a primary diagnosis of depression vs standard treatment. Subjects in treatment at two military hospitals were blinded as to study group assignment and their selfreport symptom ratings were also blinded. Quick Inventory of Depressive Symptomatology, Self-Report (QIDS-SR16) depression scores were the primary efficacy endpoint. One hundred and fifty subjects received a quantitative electroencephalography exam and were randomized to either treatment as usual or PEER-informed pharmacotherapy. Subjects in the control group were treated according to Veterans Administration/Department of Defense Guidelines, the current standard of care. In the experimental group, the attending physician received a PEER report ranking the subject's likely clinical response to on-label medications.

Results: In this post hoc interim analysis subjects were separated into Report Followed and Report Not Followed groups - based on the concordance between their subsequent treatment and PEER medication guidance. We thus evaluated the predictive validity of PEER recommendations. We found significantly greater improvements in depression scores (QIDS-SR16 $P<0.03$ ), reduction in suicidal ideation (Concise Health Risk Tracking Scale-SR7 $P<0.002$ ), and post-traumatic stress disorder (PTSD) score improvement (PTSD Checklist Military/Civilian $P<0.04)$ for subjects treated with PEER-recommended medications compared to those who did not follow PEER recommendations.

Conclusion: This interim analysis suggests that an objective tool such as PEER Interactive can help improve medication selection. Consistent with results of earlier studies, it supports the hypothesis that PEER-guided treatment offers distinct advantages over the current standard of care.

Keywords: EEG, depression, antidepressant, suicide, predictive analytics

\section{Introduction}

The authors are reporting early results from a prospective randomized controlled trial of the use of Psychiatric Electroencephalography Evaluation Registry (PEER)-guided pharmacotherapy in a military setting. The goal of the study is to demonstrate whether use of PEER-guided pharmacotherapy is 1) predictive of better clinical outcomes, including improved symptoms and reduced risk, 2) can be effectively utilized by physicians in a real-world treatment environment, and 3) can improve treatment efficiency through 
reduced trial and error. This paper addresses the first goal, presenting interim findings of a validation study in which outcomes for physicians who followed the PEER report (the "Report Followed" [RF] group) on an intent-to-treat basis are compared with outcomes for physicians who did not follow the report (the "Report Not Followed" or [RNF] group). The hypothesis is that treatment of subjects guided by an objective tool adjunctive to their physicians' clinical decision process will result in improved patient outcomes when compared to treatment based on the Veterans Administration/Department of Defense (VA/DOD) Clinical Practice Guidelines for the management of major depressive disorder (MDD). By avoiding medication classes or agents with a low likelihood of response, physicians should be able to reduce trial and error pharmacotherapy. This trial is designed to quantify this reduction and establish PEER as an efficacious method for improving the quality of medication selection.

PEER is a physician outcome registry which utilizes Quantitative Electroencephalogram (QEEG). PEER algorithms are derived from the PEER clinical registry, containing approximately 10,200 patient EEGs and 38,000 medication outcomes.

Quantitative EEG (QEEG) is the computerized processing of digitally recorded EEG to highlight specific waveform components (eg, power or frequency), transform the EEG into a format or domain that elucidates relevant information, or associate numerical results with the EEG data for subsequent review and comparison. QEEG is an accepted, well-normed, standardized test of brain electrical activity, cleared by the US Food and Drug Administration (FDA) for use by qualified medical professionals for the post hoc statistical analysis of the human EEG. Often called "neurometrics", QEEG analysis offers over 5,000 standard, validated variables which are used, alone and in combination, to classify brain electrophysiology in symptomatic and asymptomatic individuals. ${ }^{1}$ One of the virtues of this technology is that large, normative databases have been collected and validated over decades, allowing characterization of pathophysiologic abnormalities for symptomatic patients. These deviations can be expressed as statistical $z$ scores, indicating the degree of deviation from each normative variable for an individual patient. ${ }^{2}$

Sir William Osler, the great 19 th century physician, is reported to have said that the art of medicine is the art of balancing probabilities. ${ }^{3}$ With no objective, available data to individualize treatment for their patients, physicians developed the PEER to improve the quality of treatment and build evidence for care. PEER Interactive uses QEEG data to correlate known medication responders with specific patterns from the EEG in order to provide physicians with information on which drug classes and agents have a greater likelihood of benefiting a particular patient. It is modeled after a clinical registry established in the 1970s which enabled physicians treating pediatric cancer patients to share and compare patient outcomes and treatment regimens, in order to advance their knowledge of most effective treatments referenced to individual physiology. The system, which remains in place today, has dramatically improved survival rates for childhood cancers.

Similarly, research from the 1970s forward has found that antidepressants, benzodiazepines, stimulants, antipsychotics, lithium salts, and anticonvulsants induce specific QEEG changes within a few hours of dosing that are reversible upon medication withdrawal and measurable across psychiatric syndromes and in asymptomatic volunteers. A growing body of research has demonstrated strong relationships between QEEG findings and individual response to medication.

In the early 1990 s clinical researchers ${ }^{4}$ began recording medication-free EEGs on their patients. These patients were then treated according to the standard Diagnostic and Statistical Manual of Mental Disorders [DSM]-guided treatment model for the time. The researchers also painstakingly recorded patient outcomes, enabling the correlation of specific features of the EEG with known medication outcomes. They found that these correlations proved useful in a prospective clinical setting, and similar studies by other groups in recent years have confirmed these findings. ${ }^{5,6}$ This research has since been expanded upon through the systematic collection of additional EEGs and associated patient outcomes to treatment. The entire process, including receipt of the raw EEG data, correlation with known outcomes, and delivery of the findings to the referring physician and collection of patient outcomes, has since been moved to a secure web-based system known as PEER Interactive that makes this objective information available to clinicians around the world.

Building on this QEEG foundation, PEER software (Figure 1A) compares the $z$ scores of a given patient to its registry of physician outcomes, generating a probability statement for the likelihood of a positive outcome with the most commonly used medication classes and agents. The treating psychiatrist can access a PEER report online (Figure 1B), viewing a two-page display of probability of response to different medication classes and agents, before pharmacotherapy is initiated (Figure 2). The goal is to enable the clinician to avoid medication classes or agents with a low likelihood of response, thus reducing "trial and error" pharmacotherapy.

At present, PEER algorithms do not provide any prospective information for physicians related to side effects. However, the current trial builds on previous trials 
A

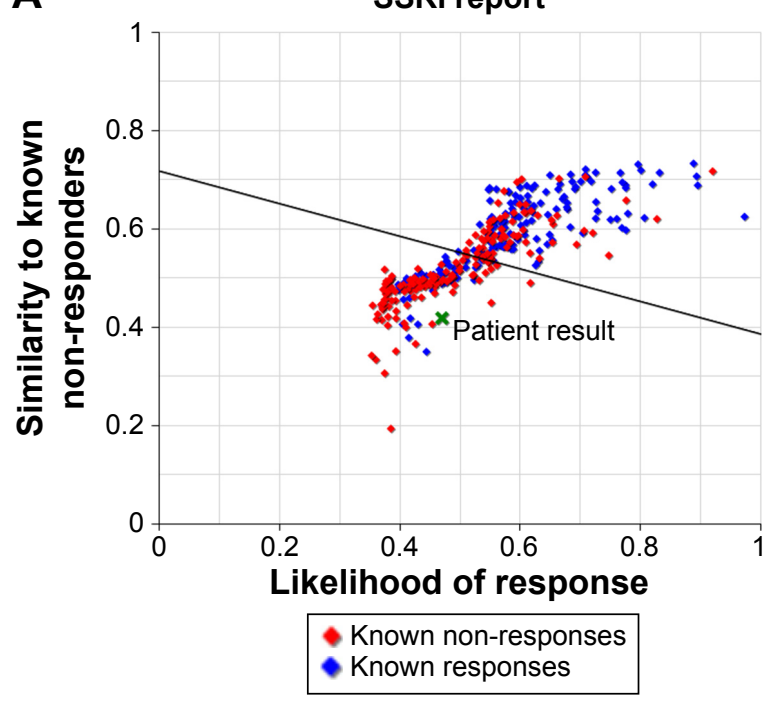

B

\section{Summary of drugs in the SSRI class}

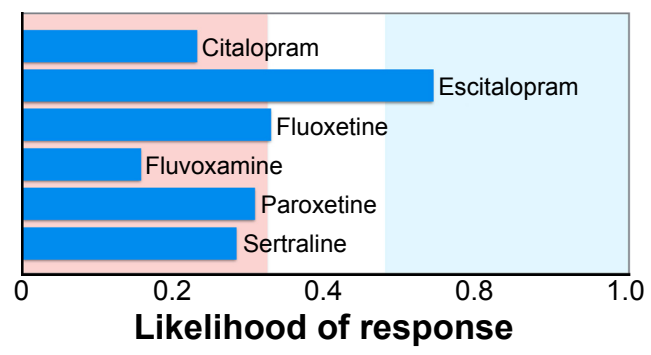

Correlation model information

Favorable response is more likely

Favorable response is less likely

Figure I (A) An example of PEER outcomes; (B) an example of a PEER SSRI report.

Abbreviations: PEER, Psychiatric Electroencephalography Evaluation Registry; SSRI, selective serotonin reuptake inhibitor.

by including endpoints for suicidality ${ }^{7}$ and serious adverse events, ${ }^{8}$ which suggests PEER can be useful in minimizing treatment emergent side effects.

With eight clinical trials $(n=830)$ demonstrating the efficacy of these predictive algorithms, the Walter Reed PEER
Interactive Trial represents the fourth randomized controlled trial of this technology. For example, earlier studies ${ }^{4,7,8}$ have suggested that use of referenced EEG, an earlier version of PEER, to guide medication selection improves efficacy, diminishes suicidality, and improves continuation

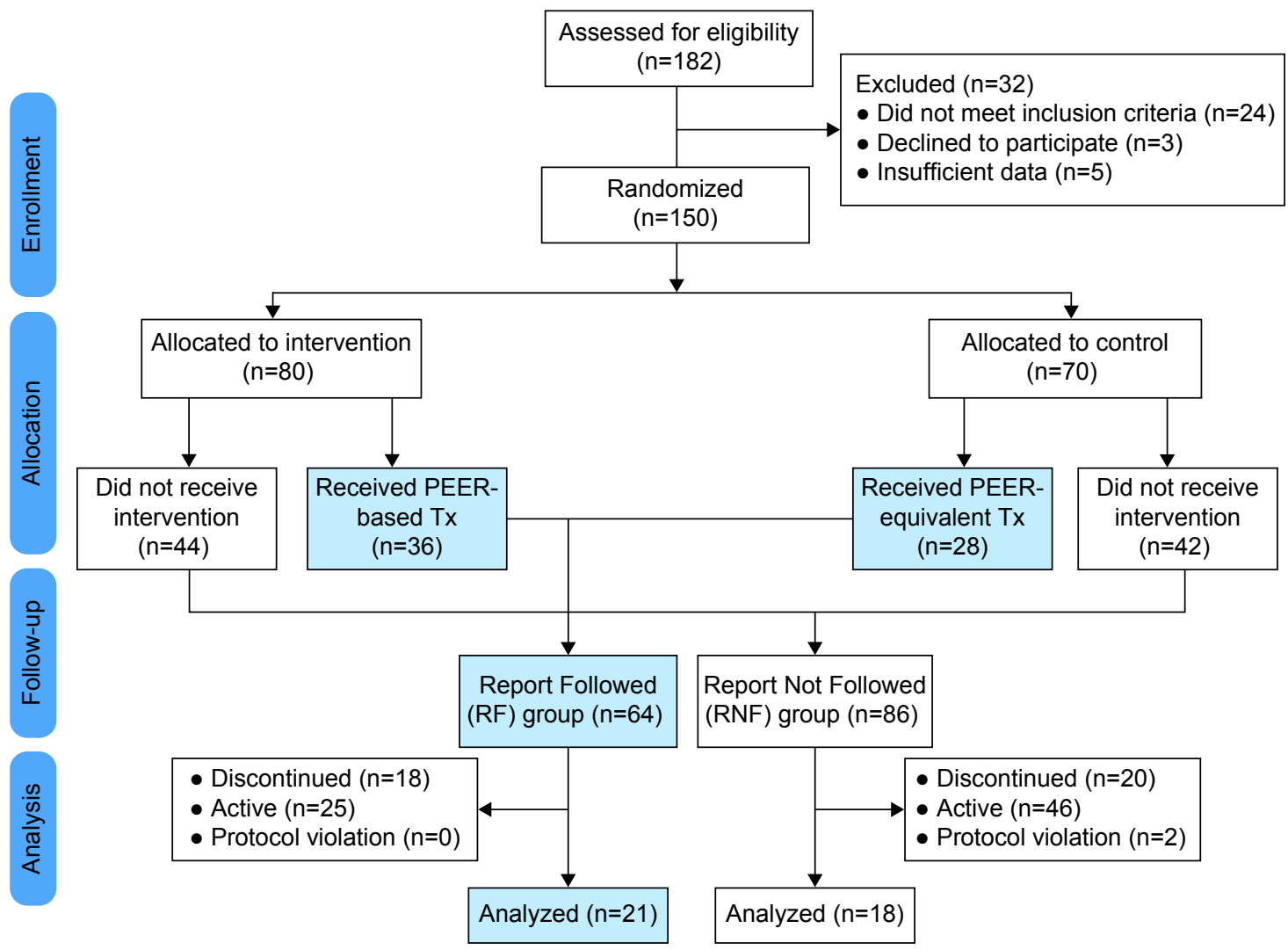

Figure 2 Patient flow diagram.

Abbreviations: PEER, Psychiatric Electroencephalography Evaluation Registry; Tx, treatment. 
of treatment. In 2010, in a prospective, multi-site study of 114 treatment refractory patients with primary depression, DeBattista et al reported significant efficacy improvement (Quick Inventory of Depressive Symptomatology, SelfReport [QIDS-SR16] $P=0.0002$ ) using referenced EEG. ${ }^{8}$ The authors concluded that, if replicated, it would represent "an easy, relatively inexpensive, predictive, objective office procedure that builds upon clinical judgment to guide antidepressant medication choice". ${ }^{8}$ In 2012, a retrospective study of 435 commercial health plan patients showed statistically significant improvements in efficacy (Clinical Global Impressions - Improvement [CGI-I] $P=0.001$ ), reduction in suicide risk $(P=0.001)$, reduced medication use $(P=0.001)$, and more rapid improvement for PEER-guided subjects than for patients whose treatment did not adhere to the PEER report. ${ }^{7}$

Over 100 independent studies using similar technologies based on QEEG have produced positive results in predicting medication response.

\section{Material and methods Method}

This is a prospective, randomized, double blind design conducted at multiple sites to evaluate the effectiveness of treatment recommendations from the PEER. We present here an interim post hoc analysis which compares outcomes for subjects treated according to PEER report recommendations (the RF group) vs subjects whose treatment is discordant with the PEER report (the RNF group). The clinical protocol was reviewed by the FDA and classified as an Investigational Device Exemption (IDE) exempt, non-significant risk study.

Study participants were male and female active duty service members, dependents, and veterans between the ages of 18-65 years. All subjects signed an informed consent and were treated under Walter Reed Human Subject Master Protocol number 378604. All participants had a primary DSM-IV diagnosis of a depressive disorder, and could also have experienced comorbid non-psychotic conditions such as post-traumatic stress disorder (PTSD). In this study, the patient was both the recipient of the study intervention and the rater who was measuring the primary outcome (QIDSSR16). Subjects were blind to study group assignment, ie, blinded to the intervention received; therefore both subjects and their ratings were blind.

The trial is designed to generate real-world evidence, so the protocol has a longer duration and is more inclusive than typical 6-8-week medication trials. ${ }^{9}$ Subjects are enrolled for up to 6 months of treatment, with minimal exclusion criteria (ie, patients can be enrolled with suicidal ideation, multiple comorbid diagnoses, and treatment resistance). For example, subjects were not excluded if they presented with active suicidality, a typical exclusion criterion in psychotropic clinical trials. While inclusive eligibility may add confounders, this was controlled for explicitly by our study design (random allocation of patients to treatment vs control arms of the study). With adequate projected sample sizes (which we have achieved, and which will continue to grow between now and study completion), this design balances all confounders - known or unknown, measured or unmeasured, measurable or unmeasurable. Thus, we have controlled for all confounders. We will only have the ability to quantify or stratify by the confounders that were measured (which is true for all randomized controlled trials), but we believe our combination of broad inclusion criteria, randomized design, and measurement of key variables using validated scales/instruments strikes the desirable balance between methodologic control of bias and confounding, and generalizability of the results for clinical practice.

A key potential advantage for PEER is that its correlations of likely response are tied to longer term outcomes with realworld psychiatric patients and their common comorbidities, since the exclusion criteria used in developing the PEER were limited to medically relevant neurological conditions (eg, seizure disorder, closed head injury, etc) or a psychotic disorder as the patient's primary diagnosis. The overall recruitment goal for this study is 1,922 subjects. The approved protocol calls for interim analyses to be performed at approximately $10 \%, 25 \%$, and $50 \%$ of expected enrollees.

The schedule of visits, procedures, and measurements is defined by the protocol as follows, in order to provide consistent data for both the control and experimental groups. All subjects received a pre-treatment EEG, a 30-minute resting eyes-closed test using standard EEG collection (with the 10-20 international set-up). EEGs were analyzed using the PEER methodology and software supplied by MYND Analytics, Inc. (Mission Viejo, CA, USA). Those enrolling who were currently using psychoactive medications discontinued use of those medications for five half-lives before their pre-treatment EEG (ie, medication washout), in order to have an "unmedicated brain" for comparison to normative databases.

All subjects will have a maximum of nine study visits, beginning with a screening visit to evaluate the appropriateness of the subject to enroll in the study. Subjects who are taking psychoactive medications at the screening visit will discontinue those medications for five half-lives before their pre-treatment EEG (ie, medication washout). At the subsequent baseline visit we will record from all subjects 
a medication-free EEG, required for computing the PEER Interactive report. Following the baseline visit, subjects will be evaluated for efficacy and safety endpoints over a series of follow-up visits. The first follow-up visit is 2 weeks after the baseline visit and the second follow-up visit is 2 weeks later, approximately 1 month following the baseline visit. All subsequent follow-up visits are every month for the next 5 months, for a total of 6 months follow-up, or until the investigator determines the subject has reached their maximum medical improvement, whichever comes first.

At every visit subjects will complete self-report forms, including QIDS-SR16, PTSD Checklist Military/Civilian (PCL-M/C), and Concise Health Risk Tracking Scale - Self Report (CHRT-7SR). The primary endpoint for this study is QIDS-SR16 percent mean change from baseline, and secondary endpoints include suicidal ideation (CHRT-7SR score percent mean change) and PTSD symptoms (PCL-M/C score percent mean change).

For subjects randomized to the active arm of the study, the intervention is information (PEER) provided to their clinician. Figure 1B shows a typical PEER Interactive report highlighting the favorable/unfavorable response prediction based on outcomes for neurophysiologically similar patients to a range of medication classes and individual agents. Physicians in the active arm were permitted to override guidance of the PEER report based on their clinical judgment and conditions specific to their patient; these subjects were considered not to have received the intervention (see RNF below). Subjects randomized to the control arm of the study received standard of care treatment based on the VA/DOD Clinical Practice Guidelines for the management of MDD.

\section{Measures}

- QIDS-SR16 measures 16 factors across nine different criterion domains for major depression. ${ }^{10}$

- CHRT-7SR, a seven-question self-report questionnaire that assesses suicidal risk of patients in clinical practice. ${ }^{11}$

- PCL-M/C, a 17 question self-reported measure of the DSM-IV symptoms of PTSD. ${ }^{12}$

- The RF and RNF designations were determined by a computer algorithm which analyzed, independent of treatment outcome, the concordance between the pharmacological treatment provided to each subject and the PEER report for the same subject. To be considered as RF the algorithm requires use of study medications from the PEER report, avoidance of non-recommended medication classes or agents, and continued use of PEER for subsequent treatments when necessary. Treatments which do not meet these criteria are considered RNF. The algorithm evaluated the treatment of subjects who had a PEER report, as well as those whose treating clinician was not provided with the PEER report but who nevertheless received pharmacotherapy which was consistent with the PEER report (Figure 2).

\section{Statistical analysis}

Demographic and clinical characteristics of study subjects at baseline were analyzed using Student's $t$-tests and chi-square tests for numerical and categorical measures, respectively. Binary outcome measures (achievement of response, yes/no) were analyzed using chi-square tests, and numerical measures (percentage change over study visits 1-6) were analyzed using repeated measures analysis of variance models. Results are presented with statistical test (chi-square or $F$-test) values, $P$-values, and 95\% confidence intervals (where applicable). All tests were performed using two-tailed alpha values of 0.05, using SPSS version 21 (IBM Corporation, Armonk, NY, USA).

\section{Results}

Of the patients enrolled, $\mathrm{N}=84(53.2 \%)$ had a diagnosis of MDD, while $\mathrm{N}=74$ (46.8\%) had diagnoses of other depressive disorders (minor depressive disorder, dysthymia, etc). Several subjects had comorbid conditions in addition to their depressive disorders; those were primarily anxiety disorders, such as Generalized Anxiety Disorder: N=32 (20.2\%), PTSD: N=30 (18.9\%), and obsessive compulsive disorder: $\mathrm{N}=2$ (1\%). Other comorbid conditions included attention deficit hyperactivity disorder $(\mathrm{N}=6,3.7 \%)$ and traumatic brain injury $(\mathrm{N}=1,0.6 \%)$.

The study groups were balanced at baseline on age, sex, and baseline QIDS-SR16, CHRT, and PTSD measures $(P$-values all $>0.05$ - see Table 1$)$. In this post hoc analysis of RF vs RNF, when PEER recommendations were followed (RF, Table 2), baseline QIDS-SR16 scores were reduced by $30 \%$ from baseline, compared with a QIDS-SR 16 reduction of only $12 \%$ for subjects whose treatment did not follow PEER (RNF). This percentage reduction from baseline represents a $144 \%$ improvement for RF vs RNF (Figure 3), with statistical significance (QIDS-SR16 $P=0.029$ ). Suicidal ideation as measure by CHRT-7SR score was 75\% better for subjects treated according to PEER than for RNF (CHRT-SR7 $P=0.0017)$. Another finding of interest (Table 2) is the 139\% improvement in change from baseline PTSD scores for patients in the RF group over the RNF group (PCL-M/C $P=0.0348$ ). Results are presented with $95 \%$ confidence intervals in Figure 4. 
Table I Demographic and clinical characteristics of study subjects

\begin{tabular}{|c|c|c|c|c|c|}
\hline Measure & Treatment arm & $\mathbf{N}$ & Mean & SD & $P$-value \\
\hline \multirow[t]{2}{*}{ Age } & Treatment & 80 & 31.76 & 10.2 & 0.764 \\
\hline & Control & 70 & 31.27 & 9.8 & \\
\hline \multirow[t]{2}{*}{ Baseline QIDS-SRI6 } & Treatment & 80 & 19.50 & 7.564 & 0.844 \\
\hline & Control & 70 & 19.26 & 7.531 & \\
\hline \multirow[t]{2}{*}{ Baseline CHRT } & Treatment & 79 & $16.5 \mid$ & 5.795 & 0.609 \\
\hline & Control & 70 & 17.01 & 6.254 & \\
\hline \multirow[t]{3}{*}{ Baseline PTSD } & Treatment & 50 & 44.50 & 14.733 & 0.301 \\
\hline & Control & 40 & 48.10 & 17.468 & \\
\hline & & Male & Female & Total & \\
\hline \multirow[t]{2}{*}{ Sex } & Treatment & 47 & 33 & 80 & 0.3810 \\
\hline & Control & 46 & 24 & 70 & \\
\hline Measure & Treatment arm & $\mathbf{N}$ & Mean & SD & $P$-value \\
\hline \multirow[t]{2}{*}{ Age } & RF & 64 & 31.79 & 11.5 & 0.921 \\
\hline & RNF & 83 & 31.62 & 9.2 & \\
\hline \multirow[t]{2}{*}{ Baseline QIDS-SRI6 } & RF & 64 & 20.66 & 6.933 & 0.071 \\
\hline & RNF & 83 & 18.42 & 7.951 & \\
\hline \multirow[t]{2}{*}{ Baseline CHRT } & RF & 64 & 17.38 & 6.012 & 0.205 \\
\hline & RNF & 82 & 16.11 & 5.877 & \\
\hline \multirow[t]{3}{*}{ Baseline PTSD } & RF & 46 & 48.00 & 15.466 & 0.268 \\
\hline & RNF & 43 & 44.19 & 16.698 & \\
\hline & & Male & Female & Total & \\
\hline \multirow[t]{2}{*}{ Sex } & RF & 37 & 27 & 64 & 0.339 \\
\hline & RNF & 55 & 28 & 83 & \\
\hline
\end{tabular}

Note: $P$-values obtained by ANOVA.

Abbreviations: ANOVA, analysis of variance; SD, standard deviation; QIDS-SRI6, Quick Inventory of Depressive Symptomatology, Self-Report; CHRT, Concise Health Risk Tracking scale; PTSD, post-traumatic stress disorder; RF, Report Followed; RNF, Report Not Followed.

While the primary focus of this paper is predictive validation based on RF vs RNF, Table S1 outlines results for these endpoints on a per protocol basis, that is, the primary treatment group vs control group analysis. In the interim analysis, per protocol baseline QIDS-SR16 scores were again reduced by $30 \%$ from baseline for subjects in the treatment group, compared with an average QIDS-SR16 reduction of $17 \%$ for subjects in the control group, though not yet a statistically significant finding (QIDS-SR16 $P=0.206$ ) due to the relatively small number of subjects who had completed their treatment at this interim analysis point $(\mathrm{n}=29)$. Per protocol suicidality findings mirrored the RF analysis

Table 2 Outcomes for subjects whose physicians followed the PEER report

\begin{tabular}{|c|c|c|c|c|c|c|c|}
\hline \multirow[t]{2}{*}{ Endpoint } & \multirow[t]{2}{*}{ Measure } & \multicolumn{3}{|c|}{ Report Followed analysis } & \multicolumn{3}{|c|}{ Test statistics } \\
\hline & & $\begin{array}{l}\text { RF\% } \\
\text { change }\end{array}$ & $\begin{array}{l}\text { RNF\% } \\
\text { change }\end{array}$ & $\begin{array}{l}\text { \% difference } \\
\text { RF vs RNF }\end{array}$ & $\mathbf{n}$ & F-test & $P$-value \\
\hline \multicolumn{8}{|l|}{ Primary efficacy } \\
\hline QIDS-SRI6 & $\begin{array}{l}\text { Mean (\%) change from } \\
\text { baseline visits I-6 }\end{array}$ & $-30 \%$ & $-12 \%$ & $144 \%$ & 39 & 5.165 & 0.029 \\
\hline \multicolumn{8}{|c|}{ Secondary endpoints } \\
\hline CHRT & $\begin{array}{l}\text { Minimum change from } \\
\text { baseline }\end{array}$ & $-24 \%$ & $-14 \%$ & $75 \%$ & 150 & 9.764 & 0.002 \\
\hline PTSD & $\begin{array}{l}\text { Minimum change from } \\
\text { baseline }\end{array}$ & $-9 \%$ & $-4 \%$ & $139 \%$ & 91 & 4.597 & 0.035 \\
\hline CGS & $\begin{array}{l}\text { Minimum change from } \\
\text { baseline }\end{array}$ & $-23 \%$ & $-13 \%$ & $68 \%$ & 145 & 5.850 & 0.017 \\
\hline CGI-physician & Minimum change & $-34 \%$ & $-22 \%$ & $57 \%$ & 150 & 9.590 & 0.002 \\
\hline CGI-patient & Minimum change & $-40 \%$ & $-22 \%$ & $82 \%$ & 150 & 17.347 & 0.0001 \\
\hline
\end{tabular}

Note: $P$-values obtained by ANOVA.

Abbreviations: ANOVA, analysis of variance; PEER, Psychiatric Electroencephalography Evaluation Registry; RF, Report Followed; RNF, Report Not Followed; QIDSSR I6, Quick Inventory of Depressive Symptomatology, Self-Report; CHRT, Concise Health Risk Tracking Scale; PTSD, post-traumatic stress disorder; CGS, Clinical Global Severity; CGI, Clinical Global Impressions. 


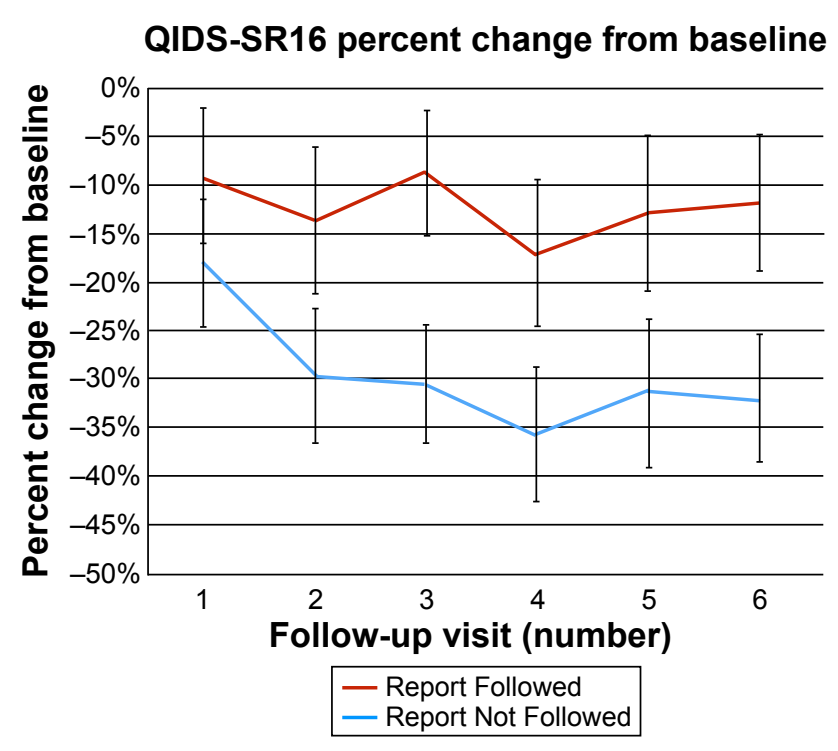

Figure 3 Primary efficacy for patients treated according to the PEER report. Abbreviations: PEER, Psychiatric Electroencephalography Evaluation Registry; QIDS-SR I6, Quick Inventory of Depressive Symptomatology, Self-Report.

with strong statistical significance - improvement in suicidal ideation was $65 \%$ greater in the treatment group - a $27 \%$ reduction in CHRT scores vs a $16 \%$ reduction in the control group, with statistical significance (CHRT-SR7 $P=0.0099)$. Similar to the RF findings, we also found statistically significant improvements in Clinical Global Severity $(P=0.028)$ and CGI-I scores - both patient $(P=0.0032)$ and physician ratings (CGI-I $P=0.0004$ ) - in favor of the treatment group. No statistically significant finding has emerged for PTSD improvement in the per protocol analysis (PCL-M/C $P=0.8743)$.

\section{Treatment adherence}

Continued participation in treatment is critical to achieving sustainable clinical outcomes, but most clinical trials experience high dropout rates after only a few weeks. "Staying on treatment can also be seen as a hard measure with little measurement error". ${ }^{13}$ In this interim analysis, the median number of follow-up visits for the RNF group was two visits, which was consistent with low retention rates reported by the military's Armed Forces Health Surveillance Center. ${ }^{14}$ The median number of visits in the RF group was five visits, suggesting a significant improvement in treatment adherence for patients in the PEER group (Figure 5). With a long duration trial (up to 6 months) compared to traditional Phase III drug trials, it is noteworthy that $40 \%$ of subjects completed the entire study in the RF group, compared with only $28 \%$ for the RNF group $(P=0.032)$.

\section{Discussion}

This interim analysis finds that subjects treated according to the PEER report experienced significantly greater improvement in depression scores (QIDS-SR16 $P=0.029$ ), suicidal ideation (CHRT-SR7 $P=0.0017$ ), and PTSD symptoms (PCL-M/C $P=0.0348$ ) compared with subjects whose clinicians did not follow PEER recommendations. The interim findings reported here provide an early confirmation of the benefit of PEER technology in assisting the clinician with selection of pharmacological treatments. These findings also suggest an extended benefit of PEER technology for improving symptoms of depression, alleviating acuity (suicidality), and decreasing dropouts (encouraging patients to comply with treatment). Our data suggest that the use of PEER technology for selecting pharmacotherapy for patients with PTSD may increase the likelihood of a favorable clinical response in this population, however more subjects are needed in the current study to confirm this finding. Our study replicates the 2010 findings of DeBattista et al (114 patient multi-site study using referenced EEG, an earlier version of PEER vs the Sequenced Treatment Alternatives to Relieve Depression [STAR*D] treatment protocol ${ }^{8}$ which
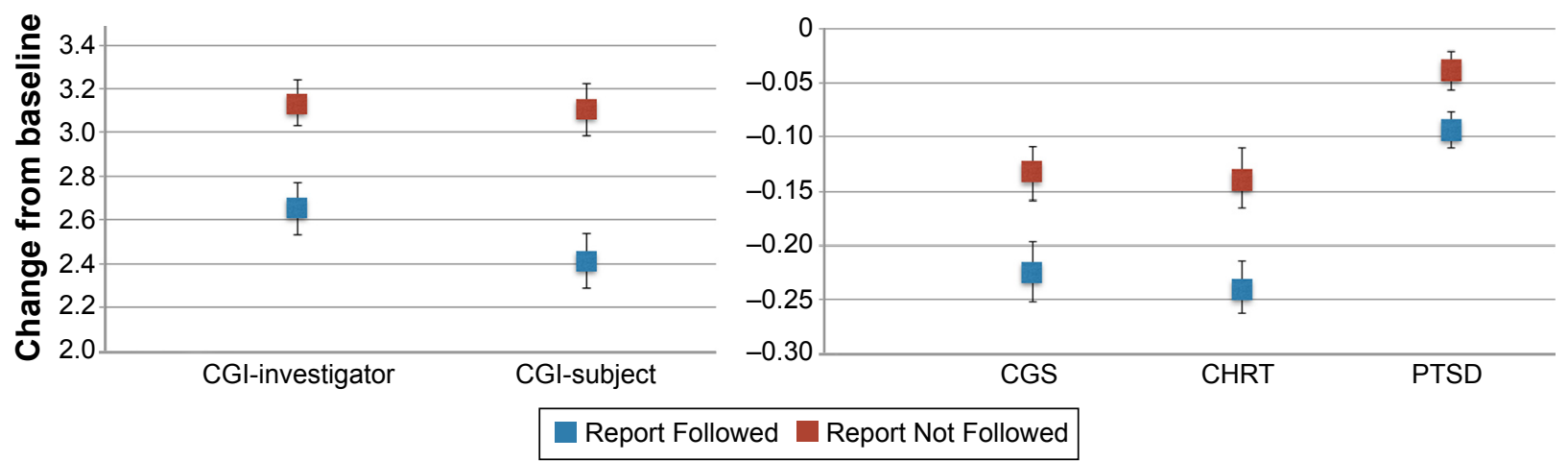

Figure $495 \%$ confidence intervals for secondary endpoints.

Abbreviations: CGI, Clinical Global Impressions; CGS, Clinical Global Severity; CHRT, Concise Health Risk Tracking Scale; PTSD, post-traumatic stress disorder. 


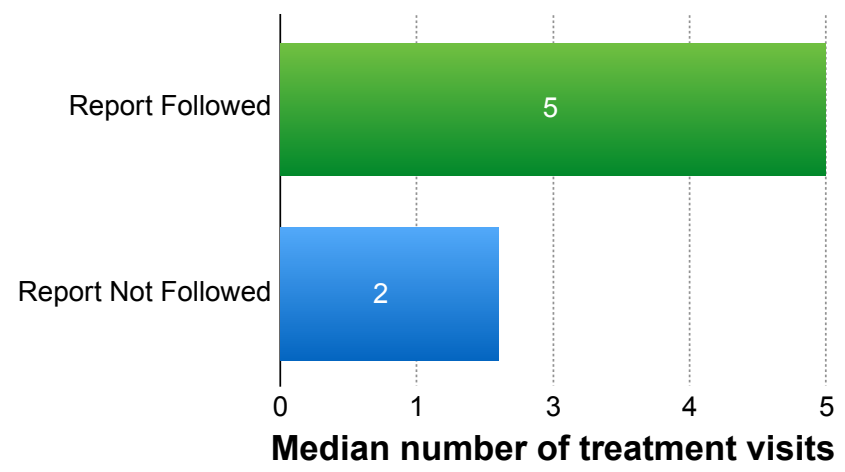

Figure 5 Participation rate by group.

highlighted the superiority of PEER-guided prescribing vs treatment as usual (based on STAR*D guidelines). This shows that the findings are consistent and reproducible across studies.

The improved adherence to treatment in the PEER group is important because the practical effect of treatment failure on the first or second drug is not simply that a patient must try a third; STAR*D and other large antidepressant trials suggested that most patients drop out after two medication failures. ${ }^{15}$ In the best conditions (eg, STAR*D), a patient's likelihood of success on a third medication was only $14 \%$, and most patients dropped out before that point. ${ }^{16}$ So, accurate selection of the first or second pharmacotherapy looms large in enhancing the odds of a patient's recovery from depression. Dropout rates in this trial were typical for a trial of such long duration. For example, in the STAR*D depression study, even the 1,085 patients who had a step-1 remission and consented to continuing care (and therefore had the highest likelihood of sustained recovery) had a relapse or dropout rate of $42.1 \%$ by the 3rd month, and $60.3 \%$ by the 6th month. Since this is a study of active military members and their dependents, there were also a significant number of dropouts resulting from transfers to new duty stations over the course of the trial.

One factor that has limited use of PEER technology in the past is the requirement of a washout period (equivalent to five half-lives of the current antidepressant medication) prior to recording the EEG. Over half of the subjects in this interim analysis required medication washout of 1 to 14 days before EEG testing. Objective analysis reveals that the concern about negative effects of washout may be overstated $^{17}$ as the washout group showed a $5.4 \%$ improvement in average QIDS-SR16 score before treatment was initiated. Overall, $52 \%$ of subjects showed an improvement in QIDSSR16 score, $22 \%$ had no change, and 26\% worsened during medication washout. There were no serious adverse events for subjects being washed out.

On a different note, the Agency for Healthcare Research and Quality has reported that second generation antidepressants have come to dominate prescribing in depression treatment, not due to superior efficacy, but due to perceived safety and improvements in tolerability. ${ }^{18}$ One notable effect is physicians' use of a broader range of medication classes such as monoamine oxidase inhibitors, tricyclics, and aminoketones (eg, bupropion) after prompts from the PEER report.

\section{Study limitations}

The Walter Reed PEER Trial has certain limitations that should be kept in mind when interpreting the study findings. First, physicians are encouraged but not required to follow the report, and are thus permitted to continue treatment as usual within the treatment group if they believe it would be best for the patient. While this does not impact the current analysis in which report followers and non-followers are compared, it reduces the number of protocol followers in the treatment group and impacts the number of evaluable subjects. On-site training sessions have been effective in increasing physician utilization of the full report (which may be better reflected in the following stages of this study). Second, because medication washout is required for any patients taking psychotropic medications at the time of enrollment, it is possible that our subjects may be different from those who declined to enroll due to the washout requirement. Finally, due to formulary and clinician preferences, a limited range of medications was used in both groups, with a strong bias toward selective serotonin reuptake inhibitor (SSRI) antidepressants. For the period of this interim analysis, approximately $60 \%$ of patients received a medication from the SSRI class, even though this class by itself has proven effective in less than $30 \%$ of patients. ${ }^{3}$ It is possible that one benefit of PEER may be in identifying alternative drugs, such as a switch to a different class drug in preference over an SSRI for subjects who had failed several SSRIs.

\section{Subsequent developments}

In April 2014 the study team was asked to provide a preliminary analysis of trial results to be forwarded to the Army Surgeon General for review by a congressional committee. In May 2014, this manuscript was prepared and submitted to Neuropsychiatric Disease and Treatment, which 
subsequently peer-reviewed and accepted it for publication. Within days of submission of this manuscript, however, the trial was placed on hold by the military sites. Subsequently, a full on-site FDA inspection of the PEER Interactive Trial was conducted in February 2016, and "revealed no significant concerns" with the trial procedures or data. Further, the research team has confirmed its findings through internal and external validation of the data included in this resubmission. Although the military has expressed its interest in continuing PEER research, at the time of this writing, no action has been taken by the military to restart the trial.

The authors note that since the inception of the Walter Reed PEER Interactive trial in 2013 over 1,500 unique patient outcomes have been added to the registry from physicians using PEER outside the military. This represents ten times the number of patients considered in this interim report; these new data are used to update the predictive classifiers at the core of the PEER report. A new multi-center study utilizing these updated classifiers and following a protocol virtually identical to the Walter Reed study presented here has been approved by Canadian Forces, and by the Western Institutional Review Board (the SMART-MD study; CNSR012).

\section{Conclusion}

Mental disorders present a profound challenge for the general population. It is imperative to validate clinical practices and technologies to improve the prescription accuracy of psychotropic medications. The interim analysis demonstrated robust early findings with statistical significance for ten of 12 endpoints (Table S1 - RF and per protocol table) including physician and patient-reported treatment efficacy (CGI-I), Suicidality (CHRT-SR7), and was approaching statistical significance for its final endpoint when halted. Results were consistent with previous randomized controlled trials, suggesting that an objective, evidence-based prescribing tool such as PEER Interactive can help clinicians meet this challenge.

\section{Acknowledgments}

The authors thank the participants of this study, the participating staff psychiatrists of the Walter Reed National Military Medical Center and Fort Belvoir Community Hospital and the members of the MYND Analytics team including COL Stewart Navarre, USMC (Ret), Chief Technology Officer Brian MacDonald, WhiteStar Consulting and Henry M Jackson Foundation support personnel. PEER Interactive utilizes Neuroguide software from Applied Neurosciences Inc.

\section{Disclosure}

Dr Neborsky serves as a medical advisor to CNS Response. He has served as paid faculty member for Dey Pharmaceuticals, Eli Lilly, Bristol Myers Squibb and has conducted investigator-initiated studies under a grant from MacNeil Pharmaceuticals.

Over the last 5 years, Dr Iosifescu has received research funding through Icahn School of Medicine at Mount Sinai from Alkermes, AstraZeneca, Brainsway, Euthymics, Neosync, Roche and Shire; he was a consultant to Avanir, Axsome, CNS Response, INSYS Therapeutics, Lundbeck, Otsuka, Servier, and Sunovion.

Dr Valuck has no conflict of interest to disclose.

\section{References}

1. Thatcher RW. Neuropsychiatry and quantitative EEG in the $21 \mathrm{st}$ Century. Neuropsychiatry. 2011;1(5):495-514.

2. Budzinsky TH, Budzinski HK, Evans JR, Abarbanel A, editors. Introduction to QEEG and Neurofeedback: Advanced Theory and Applications. 2nd ed. Academic Press; 2009.

3. Ghaemi SN, Vohringer PA, Whitham EA. Antidepressants from a public health perspective: re-examining effectiveness, suicide, and carcinogenicity. Acta Psychiatr Scand. 2013;127(2):89-93.

4. Suffin SC, Emory WH. Neurometric subgroups in attentional and affective disorders and their association with pharmacotherapeutic outcome. Clin Electroencephalogr. 1995;26(2):76-83.

5. Iosifescu DV. Electroencephalography-derived biomarkers of antidepressant response. Harv Rev Psychiatry. 2011;19(3):144-154.

6. Khodayari-Rostamabad A, Reilly JP, Hasey GM, de Bruin H, Maccrimmon DJ. A machine learning approach using EEG data to predict response to SSRI treatment for major depressive disorder. Clin Neurophysiol. 2013;124(10):1975-1985.

7. Hoffman DA, DeBattista C, Valuck RJ, Iosifescu DV. Measuring severe adverse events and medication selection using a "PEER Report" for nonpsychotic patients: a retrospective chart review. Neuropsychiatr Dis Treat. 2012;8:277-284.

8. DeBattista C, Kinrys G, Hoffman D, et al. The use of referenced-EEG (rEEG) in assisting medication selection for the treatment of depression. J Psychiatr Res. 2011;45(1):64-75.

9. Insel TR. Director's Blog: Antidepressants: A Complicated Picture; 2011 [cited December 6, 2011]. Available from: http://www.nimh.nih gov/about/director/2011/antidepressants-a-complicated-picture.shtml. Accessed August 7, 2016.

10. Ids-qids.org [homepage on the Internet]. Inventory of Depressive Symptomatology (IDS) and Quick Inventory of Depressive Symptomatology (QIDS). Available from: http://www.ids-qids.org. Accessed August 7, 2016.

11. Trivedi MH, Wisniewski SR, Morris DW, et al. Concise Health Risk Tracking scale: a brief self-report and clinician rating of suicidal risk J Clin Psychiatry. 2011;72(6):757-764.

12. Weathers FW, Litz BT, Herman DS, Huska JA, Keane TM. The PTSD Checklist (PCL): Reliability, Validity, and Diagnostic Utility. Paper presented at: the 9th Annual Conference of the International Society for Traumatic Stress Studies; October 23, 1993; San Antonio, TX.

13. Barbui C, Furukawa TA, Cipriani A. Effectiveness of paroxetine in the treatment of acute major depression in adults: a systematic re-examination of published and unpublished data from randomized trials. CMAJ. 2008;178(3):296-305 
14. Armed Forces Health Surveillance Center, Medical Surveillance Monthly Report, 2014, Vol 21(5). Available from: http://www.health. mil/Military-Health-Topics/Health-Readiness/Armed-Forces-HealthSurveillance-Branch/Reports-and-Publications/Medical-SurveillanceMonthly-Report. Accessed August 12, 2016.

15. Insel TR. Beyond Efficacy: The STAR*D trial. Am J Psychiatry. 2006; 163(1):5-7.

16. Rush AJ. STAR*D: what have we learned? Am J Psychiatry. 2007; 164(2):201-204.

17. Hoffman DA, Schiller M, Greenblatt JM, Iosifescu DV. Polypharmacy or medication washout: an old tool revisited. Neuropsychiatr Dis Treat. 2011; 7:639-648.
18. Gartlehner G, Hansen RA, Thieda P, et al. Comparative Effectiveness of Second-Generation Antidepressants in the Pharmacologic Treatment of Adult Depression. Comparative Effectiveness Review No 7. Prepared by RTI International-University of North Carolina Evidence-based Practice Center under Contract No. 290-02-0016. Rockville, MD: Agency for Healthcare Research and Quality; 2007. Available from: www.effectivehealthcare. ahrq.gov/reports/final.cfm. Accessed August 7, 2016. 


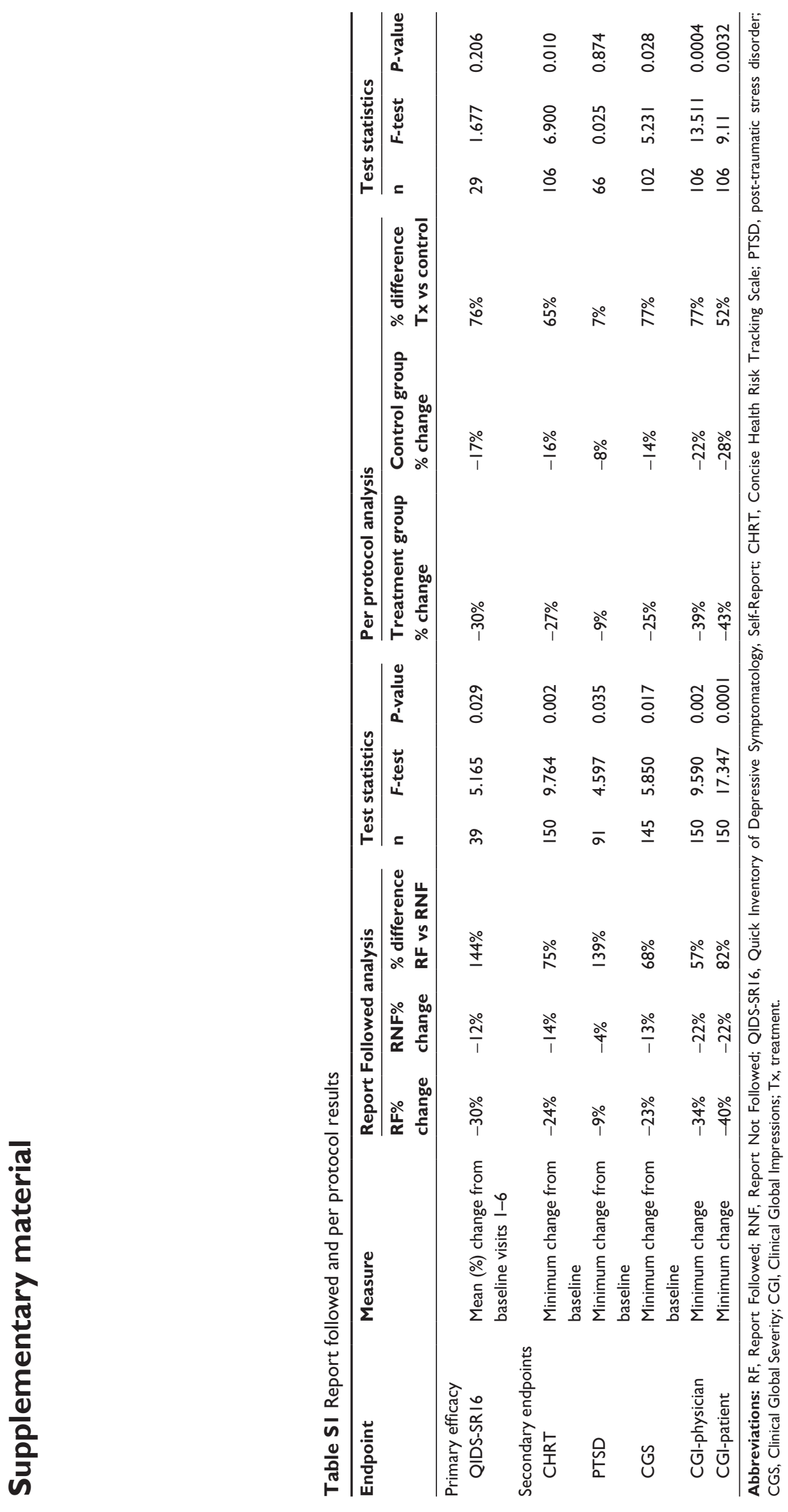




\section{Publish your work in this journal}

Neuropsychiatric Disease and Treatment is an international, peerreviewed journal of clinical therapeutics and pharmacology focusing on concise rapid reporting of clinical or pre-clinical studies on a range of neuropsychiatric and neurological disorders. This journal is indexed on PubMed Central, the 'PsycINFO' database and CAS,

and is the official journal of The International Neuropsychiatric Association (INA). The manuscript management system is completely online and includes a very quick and fair peer-review system, which is all easy to use. Visit http://www.dovepress.com/testimonials.php to read real quotes from published authors.

Submit your manuscript here: http://www.dovepress.com/neuropsychiatric-disease-and-treatment-journal 